\title{
„Mismatch beim Impfstoff wäre vermeidbar gewesen"
}

\author{
Der Impfschutz von Diabetikern gegen Grippe ist \\ mangelhaft. In der letzten Saison wurde die Situation \\ durch Probleme mit dem Standardimpfstoff ver- \\ schärft. Über die Ursachen sprach InFo Diabetologie \\ mit Prof. Klaus Wahle, Internist aus Münster.
}

Die STIKO empfiehlt, alle Diabetiker gegen Influenza zu impfen. Warum ist das so wichtig?

Wahle: Diabetiker zählen aufgrund ihrer chronischen Erkrankung ebenso wie etwa auch Asthma- und COPD-Patienten zu den Hochrisikopatienten, was Infektionskrankheiten angeht. Das Infektionsrisiko ist erhöht und die klinischen Verläufe sind in der Regel weitaus schwerer als bei gesunden Menschen.

Haben Sie den Eindruck, dass die Impfempfehlung konsequent umgesetzt wird?

Wahle: Bei Weitem nicht. Die Indikationsimpfungen der STIKO werden grundsätzlich nur unzureichend durchgeführt. Man vermutet, dass nur etwa 25\% aller Patienten, für die eine Indikationsimpfung empfohlen wird, tatsächlich geimpft werden. Man muss davon ausgehen, dass nicht einmal die Hälfte der Diabetiker unter 60 Jahren die jährliche Influenzaimpfung bekommt.

\section{Woran liegt das?}

Wahle: Ich bin, ehrlich gesagt, schon froh, wenn in den Arztpraxen die Standardimpfungen der STIKO konsequent umgesetzt werden - auch das passiert ja nicht immer. Dann ist es natürlich noch schwieriger, alle 250 Indikationsimpfungen zu überblicken und zu realisieren. Aber eigentlich müsste es sich langsam herumgesprochen haben, dass man bei den Diabetikern nicht bis zum 60. Lebensjahr mit der Grippeimpfung warten, sondern eigentlich schon mit der Diagnose beginnen muss.

Die geringen Impfquoten hängen aber auch mit der Influenzaimpfung selbst zusammen. In den vergangenen Jahren sind die Durchimpfungsraten in Deutschland nicht etwa gestiegen, sondern waren rückläufig. Als wesentlicher Grund dafür wird das Ausschreibungsverfahren der Krankenkassen angesehen, die den einzelnen Ärzten mehr oder weniger nur einen Standardimpfstoff zur Verfügung stellen. Will man darüber hinaus auch andere Impfstoffe verwenden, droht ein Regressrisiko. Die Impfung selbst ist mit einer relativ hohen Unsicherheit belastet: Welcher Impfstoff hat in dieser Saison die Ausschreibung gewonnen? Kann ich ihn in ausreichendem Umfang rechtzeitig beziehen, und woher beziehe ich ihn? Das Ganze ist also nicht leichter, sondern eher intransparenter geworden.

In der letzten Grippesaison kam ein weiteres Problem hinzu: Die Abdeckung der zirkulierenden Viren durch den Impfstoff war mangelhaft. Was war der Grund dafür?

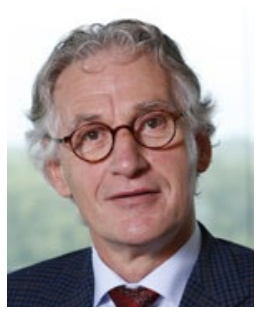

Prof. Dr. med. Klaus Wahle, Münster

Facharzt für Innere Medizin und

Allgemeinmedizin

Am Pastorenbusch 2, 48161 Münster

Wahle: Das Erschreckende ist, dass zum Ende der letzten Saison alle Experten von der Situation überrascht wurden: Es waren nicht im Wesentlichen die A-Stämme, die die Influenzaerkrankungen in Deutschland hervorgerufen haben, sondern die BStämme, und zwar vor allem die relativ neue B-Linie Victoria, die nicht im trivalenten Standardimpfstoff enthalten war, sondern nur im tetravalenten Impfstoff. Dieser Impfstoff stand zwar in Deutschland auch zur Verfügung, da er aber einen Virusstamm mehr enthält und daher auch teurer ist, hatte er im Rahmen der Ausschreibungsverfahren der Krankenkassen überhaupt keine Chance. Das heißt, wir haben fleißig geimpft, aber möglicherweise den falschen Impfstoff. Einen gewissen Mismatch gibt es jedes Jahr, aber in diesem Jahr mit dem neuen B-Stamm ist er sehr stark ausgefallen. Darunter hat die Impfstoffeffektivität drastisch gelitten. Das hätte man vermeiden können, indem man den Impfärzten die Wahl des Impfstoffs überlassen hätte.

Die WHO hat mit ihren Empfehlungen auf diesen Mismatch reagiert. Der trivalente Impfstoff für die bevorstehende Grippesaison wird nun die B-Linie Victoria enthalten. Halten Sie diese Maßnahme für ausreichend?

Wahle: Auch das ist ja nur Spekulation. Wir wissen einfach nicht, was uns in der kommenden Saison erwartet. Häufig passen Impfstoff und zirkulierende Viren relativ gut zusammen, aber nicht in jedem Fall. Ich plädiere dafür, dass ein Impfstoff mit möglichst breiter Abdeckung angeboten wird, also ein tetravalenter Impfstoff, der beide B-Linien enthält. Damit können wir momentan den sichersten Schutz erreichen.

Warum wird der tetravalente Impfstoff bislang nicht von der STIKO empfohlen?

Wahle: Die STIKO hat ihre Aufgabe bisher vor allem so verstanden, dass sie über die Impfindikationen entscheidet - sie bezieht sich nicht auf den Impfstoff als solchen. Ich glaube aber, dass die STIKO langfristig nicht bei dieser Strategie bleiben kann. Es gibt ja außer den tri- und tetravalenten Grippeimpfstoffen zum Beispiel auch noch adjuvantierte Impfstoffe, die besonders bei Älteren Vorteile haben.

Das Interview führte Dr. Beate Schuhmacher. 